\title{
An Incidentally Discovered Small and Asymptomatic Para-Aortic Paraganglioma
}

\author{
T. Yamaguchi ${ }^{\mathrm{a}} \quad$ M. Tada ${ }^{\mathrm{b}} \quad$ H. Takahashi ${ }^{\mathrm{a}} \quad$ R. Kagawa ${ }^{\mathrm{a}} \quad$ R. Takeda ${ }^{\mathrm{a}} \quad$ S. Sakata ${ }^{\mathrm{a}}$ \\ M. Yamamoto ${ }^{a} \quad$ D. Nishizaki ${ }^{a}$

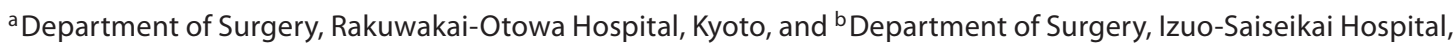 \\ Osaka, Japan
}

\section{Key Words}

Para-aortic paraganglioma $\cdot$ Asymptomatic paraganglioma

\begin{abstract}
Preoperative diagnosis of asymptomatic paraganglioma is difficult due to the lack of specific symptoms. In this report, we present a rare case of a small and asymptomatic paraaortic paraganglioma. A 34-year-old woman who complained of back pain was admitted for further examination. No abnormal findings were observed on physical or laboratory examinations. An abdominal CT scan and an abdominal MRI incidentally noted a mass about $3 \mathrm{~cm}$ in diameter adjacent to the right edge of the inferior vena cava. The following aortic angiography showed the tumor with a feeding artery diverting directly from the aorta. The tumor was completely resected by laparotomy. The resected tumor, $3 \times 3 \times 3 \mathrm{~cm}$ in size, was soft, dark-reddish and encapsulated. Immunohistochemical examinations showed that it was positive for neuron-specific enolase, chromogranin A and adrenocorticotropin. Under these findings, the diagnosis of para-aortic paraganglioma was determined. Seven years after the operation, she remains asymptomatic and free of disease.
\end{abstract}

Copyright $\odot 2008$ S. Karger AG, Basel

\section{Introduction}

Paraganglioma is a rare tumor, which arises from extra-adrenal chromaffin cells and represents $10-18 \%$ of all chromaffin tissue-related tumors [1]. Most paragangliomas are located in the head and neck [1]. Para-aortic paraganglioma is an extremely rare disease. Many cases of clinically asymptomatic paragangliomas are not found until they have grown comparatively large. In this report, we present a case of a small and asymptomatic para-aortic paraganglioma, which was completely removed by surgery.

\section{Case Report}

A 34-year-old woman with back pain was admitted for further examination in March 2000. On physical examination, she exhibited a blood pressure of $108 / 68 \mathrm{~mm} \mathrm{Hg}$ and a regular sinus cardiac rhythm of $68 / \mathrm{min}$. She was slender and no clinical symptoms for Cushing's syndrome or aldosteronism were observed. No abnormal findings were observed on physical and laboratory examinations including tumor markers (CEA $0.5 \mathrm{mg} / \mathrm{ml}$, CA19-9 $28 \mathrm{IU} / \mathrm{ml}$ and NSE $5.4 \mathrm{mg} / \mathrm{ml})$. Her back pain disappeared after admission. Because it disappeared by bed rest, it was thought to be a muscular pain due to overwork. However, an abdominal CT scan incidentally noted a ring-enhanced mass $3 \mathrm{~cm}$ in diameter adjacent to the right edge of the inferior vena cava. The mass was located at the level of the $\mathrm{L}_{3}-\mathrm{L}_{4}$ intervertebral space. It was shown
Tetsuya Yamaguchi, MD

Department of Surgery, Rakuwakai-Otowa Hospital

2 Otowachinjicho, Yamashina-ku

Kyoto 607-8062 (Japan)

Tel. +81 75593 4111, Fax +81 75581 6935, E-Mail rakuwadr011@rakuwadr.com 


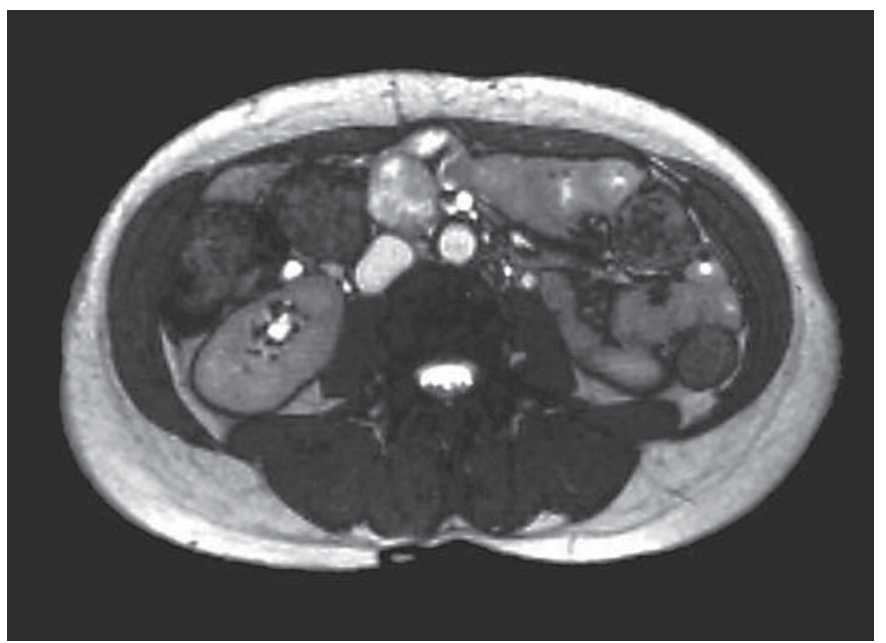

Fig. 1. Abdominal MRI with gadolinium showed a heterogeneously enhanced mass.

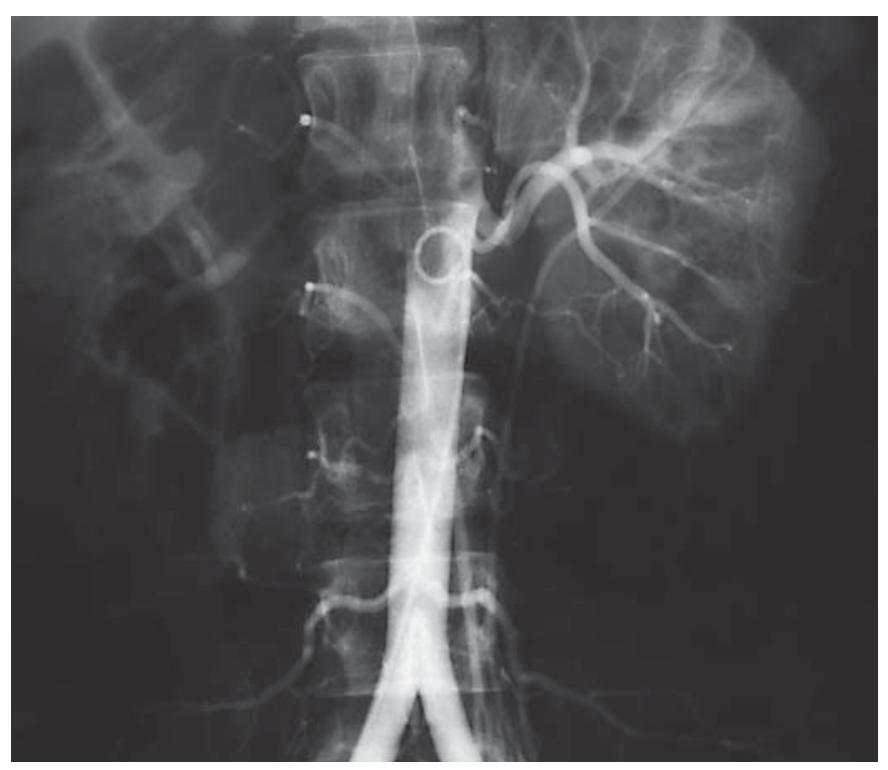

Fig. 2. Aortography showed a tumor that was well vascularized at the level of the $\mathrm{L}_{3}-\mathrm{L}_{4}$ intervertebral space.

on MRI with gadolinium as a heterogeneously enhanced mass (fig. 1). Aortography showed that the tumor had a feeding artery directly diverting from the aorta (fig. 2). Gallium scintigraphy revealed no remarkable accumulation. Although the tumor was considered to be asymptomatic, its malignant potential could not completely be ruled out. Therefore, under the diagnosis of paraaortic tumor with unknown malignant potential, she underwent laparotomy. On laparotomy, a solid mass $3 \mathrm{~cm}$ in diameter was found, which was located at the right side of the 3rd lumbar ver-
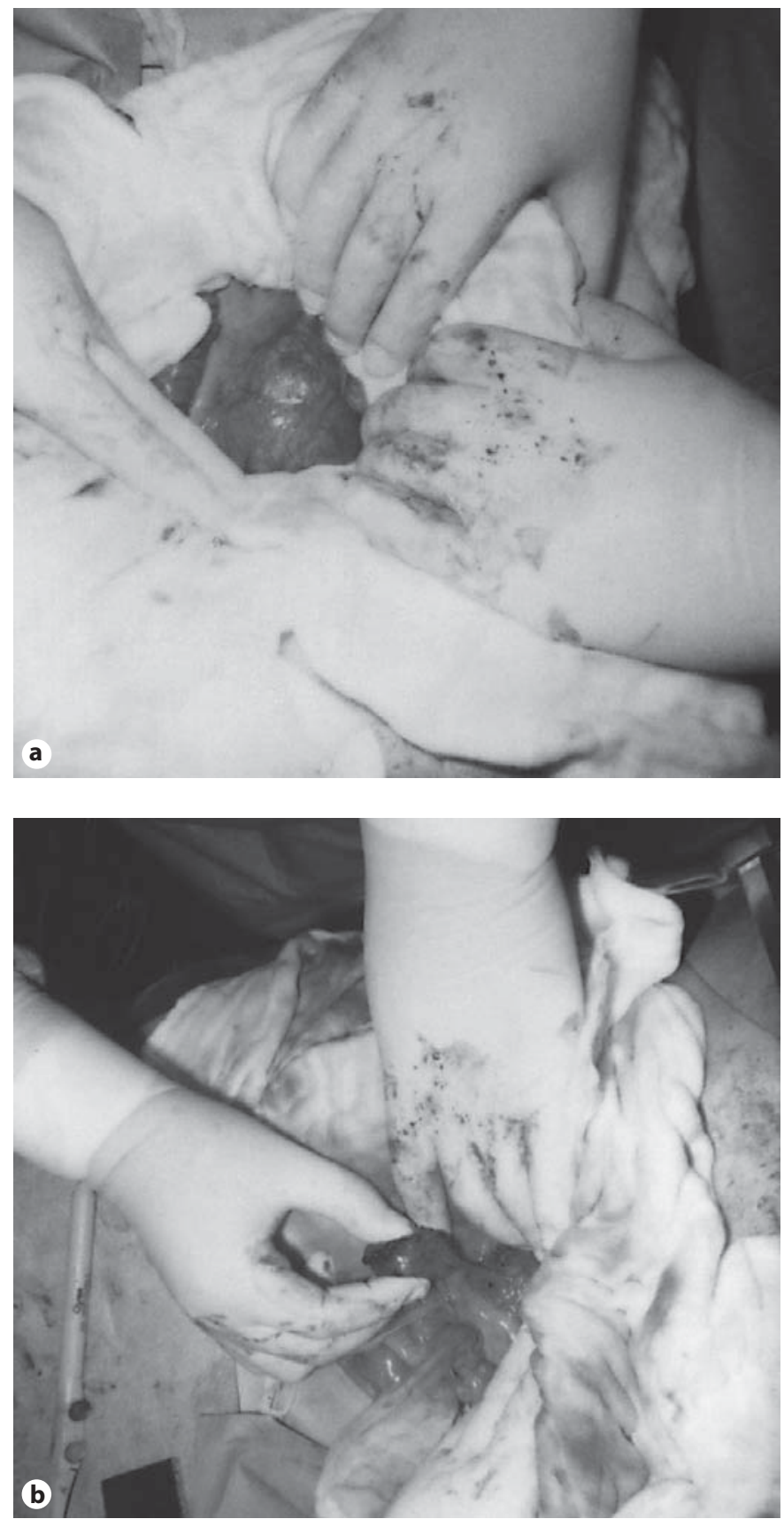

Fig. 3. a The mass, $3 \mathrm{~cm}$ in diameter, revealed no adhesion or invasion into the surrounding tissues. $\mathbf{b}$ The mass was dissected and the feeding artery was isolated.

tebral bone. It revealed neither adhesion nor invasion into the surrounding tissues (fig. 3a). In addition, no other tumors nor lymphadenopathies were found in the peritoneal cavity. The tumor was dissected and the feeding artery was isolated. By the transection of the feeding artery, the tumor was completely re- 

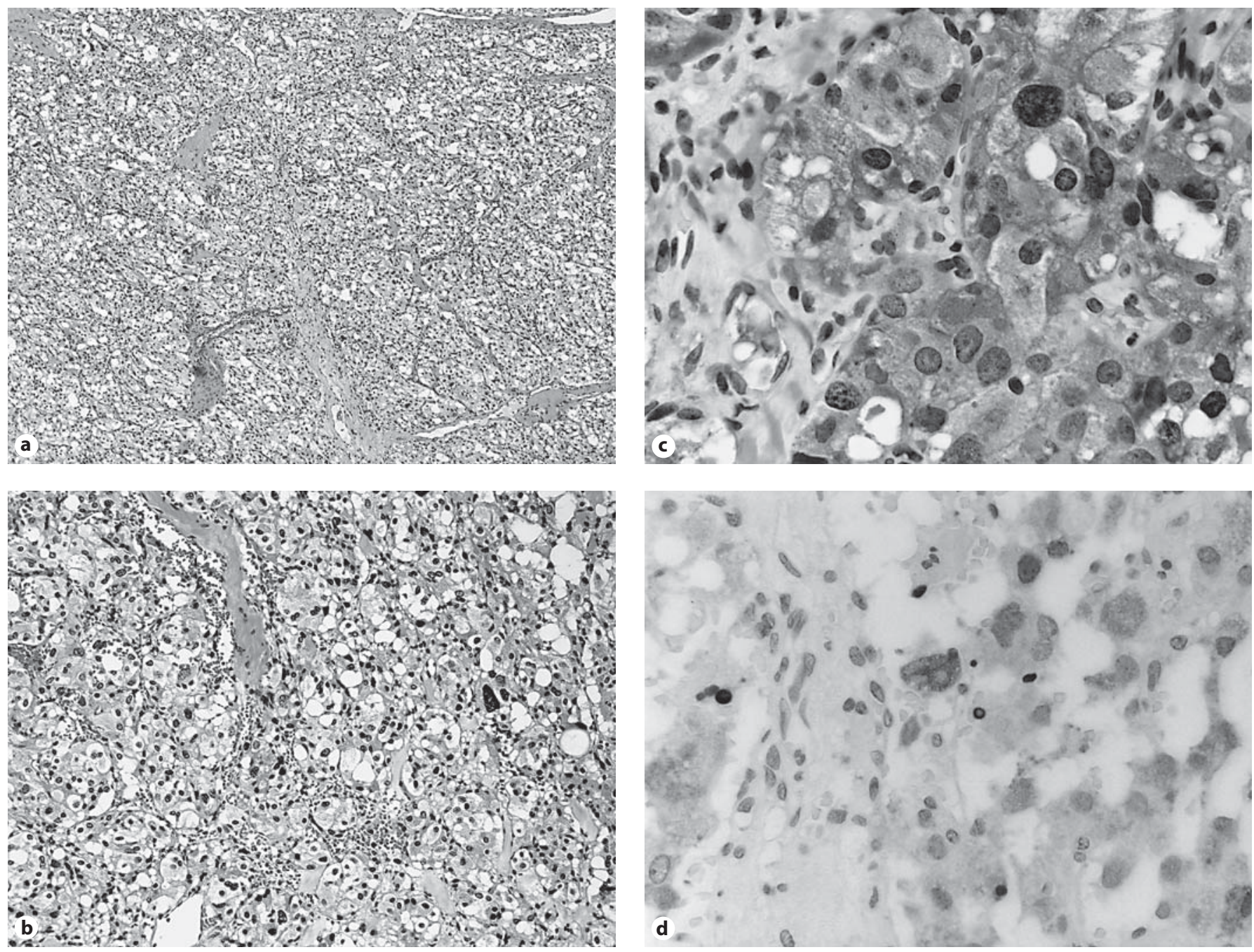

Fig. 4. a HE $\times 40$. Tumor cells of palely eosinophilic and finely granular cytoplasm with round to oval small nuclei arranged in an anastomosing trabecular pattern. The stroma is composed of a prominent vascular network. b HE $\times 200$. A moderate degree of nuclear pleomorphism is occasionally seen, but mitotic figures are inconspicuous. $\mathbf{c} \times 400$. Cytoplasmic staining with chromogranin A. $\mathbf{d} \times 400$. Cytoplasmic staining with adrenocorticotropin.

sected (fig. 3b). During the operation, her blood pressure and heart rate were quite stable. The resected tumor, $3 \times 3 \times 3 \mathrm{~cm}$ in size, was soft, dark-reddish and encapsulated. In cross section, the well-circumscribed tumor showed foci of hemorrhage without necrosis. Microscopically, the tumor was composed of large polygonal cells arranged in an anastomosing trabecular to alveolar pattern separated by a delicate vascular network (fig. 4a). Tumor cells mostly had palely eosinophilic and finely granular abundant cytoplasm with small round nuclei. A moderate degree of nuclear pleomorphism was occasionally observed, but mitotic figures were infrequent (fig. 4b). Immunohistochemically, tumor cells were positive for neuron-specific enolase, synaptophysin, chromogranin A and adrenocorticotropin (fig. 4c, d). Finally, it was diagnosed as an extra-adrenal paraganglioma. Her postoperative course was uneventful, and she was discharged 7 days after the operation. Thereafter, she has been followed by an annual screening with an abdominal CT scan. Seven years after the operation, she remains asymptomatic and free of disease.

\section{Discussion}

Paragangliomas are rare endocrine tumors that arise from paraganglionic tissue and the mean age at diagnosis is reported to be 47 years old [1]. In most cases, they are discovered and diagnosed incidentally. Paragangliomas 
are groups of cytochemically and morphologically similar cells derived from the neural crest. They include such tissues as the adrenal medulla, carotid and aortic bodies, organs of Zuckerkandl, and other unnamed paraganglia occurring in the distribution of the sympathetic and parasympathetic nerves [2]. The physiologic role of extraadrenal paraganglia in the retroperitoneum is assumed to be the homeostatic maintenance of blood pressure in early human life [3]. By means of the degree of affinity of the tumor cells for chromium, paragangliomas are classified into two groups: chromaffin and nonchromaffin. Paragangliomas are also classified into functional and nonfunctional by the presence of accompanying clinical symptoms such as hypertension, hyperhidrosis and hyperglycemia, which are characterized by the secretion of catecholamines. Although the tumor in this case produced ACTH, the patient did not show any accompanying clinical symptoms. The diagnosis of paragangliomas is infrequently made preoperatively unless the tumors are functional [2]. Most patients with nonfunctional tumors have pain and a mass and occasionally have symptoms of metastases. Functional tumors are easier to diagnose because urinary catecholamines are elevated, usually with a predominance of norepinephrine [2]. The determination of urinary methoxycatecholamine secretion is useful for the diagnosis of functional tumors, although it has not been determined in this case. The biochemical diagnosis of hyperfunctional paraganglioma or adrenal pheochromocytoma is based on excessive secretion of chatecholamines and their metabolites. It has been estimated that as many as $10 \%$ of paragangliomas arise outside the adrenal glands [4]. Paragangliomas of the retroperitoneum arise from dispersed paraganglia that tend to be symmetrically distributed in close relation to the aorta and sympathetic nervous system. Neoplasms arising from the aorticosympathetic paraganglia have been referred to as extra-adrenal pheochromocytomas because of their histologic, biochemical and clinical similarities to adrenal pheochromocytoma [4]. On follow-up, paragangliomas are reported to be cured in approximately $69 \%$ of patients [1]. The malignant potential of the tumor is determined by local invasion as well as distant metastases as there are no characteristic cellular changes [4]. In retroperitoneal paragangliomas, one third of the tumors are reported to be functional and $50 \%$ of the tumors are thought to be malignant [2]. By comparison, the rate of malignancy is thought to be about $10 \%$ for paragangliomas of the adrenal gland [5].

Paragangliomas of the retroperitoneum are a group of tumors that cause considerable difficulty in both diagno- sis and treatment. They occur most commonly in the para-aortic area from the origin of the inferior mesenteric artery to the aortic bifurcation [4]. An accurate preoperative clinical diagnosis of paraganglioma is seldom made unless there are overt symptoms related to excess catecholamine secretion. Angiography has been replaced by CT scanning for initial evaluation of retroperitoneal and abdominal masses but may still be helpful in revealing vascular invasion or in demonstrating small metastases [6]. Although CT scan is also the first modality used to localize tumors, metaiodobenzylguanidine (MIBG) scans may be used to delineate multiple tumors and small tumors not seen on CT scan [7]. The role of MIBG in nonfunctional tumors is unclear [2]. MRI is associated with the lowest false-negative localization rate, whereas MIBG scintigraphy is the least sensitive imaging study [1]. However, MIBG scintigraphy is highly specific and may be the only positive imaging test in some patients. Because of the rarity of these tumors, natural history is not always clear and treatment protocols have not been established. Aggressive surgery is mandatory to obtain disease-free survival with low morbidity and mortality. Once the diagnosis of paraganglioma is made, every attempt should be made to perform a complete surgical resection. During surgery, attention has to be paid to the endocrinological characteristics of the paraganglioma. Clinically and biochemically silent paragangliomas may cause hemodynamic instability when they are manipulated during surgery, and low-dose $\alpha$-adrenergic blockade should be considered in most patients [1]. In the case of large tumors, because of an abundant blood supply to the tumor, hemostatic procedures are thought to be very difficult. In those cases, a preoperative angiography, which confirms the relationship between the great vessels and the tumor vessels, is also considered to be very useful. If a tumor is felt to be unresectable at surgery, attempts to reduce its size by chemotherapy or radiation or embolization may be indicated because resection offers the only chance to cure. Approximately one third of patients have persistent or recurrent paragangliomas [1]. Since the malignant potential of paragangliomas cannot always be definitely judged from pathological findings, regardless of the pathological diagnosis, many cases of paragangliomas necessitate a long-term follow-up.

In conclusion, we reported a rare case of a small and asymptomatic para-aortic paraganglioma that was completely removed by surgery.

Eur Surg Res 2008;40:14-18 


\section{References}

-1 Erickson D, Kudva YC, Ebersold MJ, Thompson GB, Grant CS, Heerden JA, Young WF: Benign paragangliomas: clinical presentation and treatment outcomes in 236 patients. J Clin Endocrinol Metab 2001;86:52105216.

-2 Sclafani LM, Woodruff JM, Brennan MF: Extra-adrenal retroperitoneal paragangliomas: nature history and response to treatment. Surgery 1990;108:1124-1130.
-3 Lack EE, Cubilla AL, Woodruff JM, Lieberman PH: Extra-adrenal paragangliomas of the retroperitoneum: a clinicopathologic study of 12 tumors. Am J Surg Pathol 1980;4: 109-120.

-4 Somasundar P, Krouse R, Hostetter R, Vaughan R, Covey T: Paragangliomas - A decade of clinical experience. J Surg Oncol 2000;74:286-290.

5 Scott HW, Dean RH, Oates JA, Robertson D, Rhamy RK, Page DL: Surgical management of pheochromocytoma. Am Surg 1981;47:813.
6 Patel YD, Morehouse HT: Malignant paragangliomas of the retroperitoneum: value of computed tomography and angiography. Clin Radiol 1984;35:185-188.

7 Chatal JF, Charbonnel B: Comparison of iodobenzylguanidine imaging with computed tomography in locating pheochromocytoma. J Clin Endocrinol Metab 1985;61:796872 . 